
\title{
25 Research Soure \\ SY-707, an ALK/FAK/IGF1R Inhibitor, Suppresses Growth and Metastasis of Breast Cancer Cells
}

Ping Liu

Capital Medical University

Yinghui Sun

Shouyao Holding

Shuang Liu

Shouyao Holdings

Jing Niu

Capital Medical University

Xijie Liu

Shouyao Holdings

Binghui Li

Capital Medical University

Qiaoyun Chu ( $\nabla$ qychu@ccmu.edu.cn )

Capital Medical University https://orcid.org/0000-0002-7344-0395

Primary research

Keywords: SY-707, anti-tumor, anti-metastasis, breast cancer

Posted Date: February 10th, 2021

DOI: https://doi.org/10.21203/rs.3.rs-148993/v2

License: (c) (i) This work is licensed under a Creative Commons Attribution 4.0 International License.

Read Full License

Version of Record: A version of this preprint was published at Acta Biochimica et Biophysica Sinica on February 1st, 2022. See the published version at https://doi.org/10.3724/abbs.2022008. 


\section{Abstract}

Background Focal adhesion kinase (FAK), a multi-functional cytoplasmic tyrosine kinase, plays a critical role in cancer migration, proliferation and metastasis via regulating multiple signaling pathways. SY-707 is an ALK/FAK/IGF1R multi-kinase inhibitor and now evaluated in Phase II clinical trial for ALK positive non-small cell lung cancer (NSCLC).

Methods HTRF (Homogeneous Time-Resolved Fluorescence) assay was used to analyze kinase enzyme activity to determine inhibitory activities of SY-707 on other kinases. ATP content, PE-Annexin V and would healing assays were used to examine cell proliferation, cell cycle and migration when cells were treated with SY707. Then, SD rat and beagle dog models were used to evaluate the pharmacokinetics profile, and mouse xenograft model was used to evaluate the in vivo anti-cancer activities of SY707.

Results In this study, we assessed preclinical anti-growth and anti-metastasis potency of SY-707 in breast cancer cells. SY-707 was able to inhibit the growth of breast cancer cell lines and induced cell apoptosis by suppressing the FAK signaling pathways. Moreover, SY-707 exerted inhibition on cell migration and adhesion in a dose-dependent manner. In T47D xenograft mice, SY-707 had significant anti-tumor activities lonely or synergistically with Paclitaxel. Meanwhile, SY-707 also displayed significant suppression on spontaneous metastasis of tumor to the lung in $4 \mathrm{~T} 1$ murine breast cancer xenograft model.

Conclusions SY-707 illustrated potent anti-proliferation and anti-migration potential in breast cancer in vitro and in vivo, implying its therapeutic application for the treatment of breast cancer in future clinical trials.

\section{Introduction}

Breast cancer is the mostly common diagnosed cancer for women worldwide. It is estimated that more than 1.4 million women worldwide are diagnosed with breast cancer, and over 450,000 women will die from this disease[1]. Treatment of breast cancer is particularly difficult when cancer metastasis occurs in other organs spread from primary lesions[2]. In the past several decades, although new diagnostic, prognostic and therapeutic strategies of breast cancer were developed, the survival rate for breast cancer patients with metastatic disease has not changed significantly[3].

FAK, an intracellular non-receptor protein tyrosine kinase with a molecular weight of $125 \mathrm{kDa}$, performs its biological functions by interacting with multiple cytokine factors such as integrins, IL-4, VCAM-1, as well as growth factor receptors like epithelial growth factor receptor (EGFR), vascular endothelial growth factor receptor (VEGFR), and platelet-derived growth factor receptor (PDGFR)[4]. FAK also worked as a nuclear protein to promote degradation of oncogene proteins p53 via ubiquitination and enhanced cell proliferation and reduced inflammatory responses[5]. Moreover, FAK has been implicated in the development of breast cancer and other malignancies. High level of FAK expression was observed in aggressive breast cancer[6, 7], and only low expression of FAK protein was detected in normal human 
breast tissue and para-carcinoma tissue[8]. Thus, FAK can be a marker of malignant transformation and a prognostic indicator in breast cancer. Recently, accumulated evidence supported FAK as a therapeutical target for cancer treatment [9-12] and several FAK inhibitors including PF-562271[13, 14] and TAE226 [15]were approved to clinical studies. However, no positive clinical results were released and efficacious FAK inhibitors were expected for further development[16].

SY-707 (previous known as CT-707) is a multiple kinase inhibitor against ALK, FAK and IFG1R, and had been approved for treatment of ALK positive NSCLC in 2016 (NCT02695550). In this study, we have analyzed the anti-tumor and anti-metastasis effects of SY-707 in breast cancer in vitro and in vivo, and the results were supported to expand additional clinical indications in the future.

\section{Materials And Methods}

Compound synthesis. SY-707, [N-isopropyl-2-((2-((2-methoxy-4-(4-(4-methylpiperazin-1-yl) piperidin-1yl)phenyl)amino)-6,7-dihydro-5H-pyrrolo[2,3-d]pyrimidin-4-yl)amino)benzenesulfonamide] was designed and synthesized by Centaurus BioPharma Co., Ltd. PF-562271 was synthesized in house. Compounds were stocked at $10 \mathrm{mM}$ concentration in 100\% DMSO and diluted for biological test prior to use in each experiment.

Kinase enzyme assay. ALK, Pyk2 and FAK enzymes were purified with in sf9 insect cells, and HTRF ${ }^{\circledR}$ KinEASE ${ }^{T M}$-TK was obtained from Cisbio Bioassays. HTRF (Homogeneous Time-Resolved Fluorescence) assay was used to analyze kinase enzyme activity and compound potency. Compounds were serially diluted in 3 folds from $1 \mathrm{mM}$ stock solution in DMSO for 11 points. $4 \mu \mathrm{L}$ of the diluted solution of the compounds was added to $96 \mu \mathrm{L}$ of reaction buffer $\$ 50 \mathrm{mM} \mathrm{HEPES} p H 7.0,0.1 \mathrm{mM} \mathrm{Na}_{3} \mathrm{VO}_{4}, 0.01 \% \mathrm{BAS}, 5$ $\mathrm{mM} \mathrm{MgCl}_{2}, 1 \mathrm{mM} \mathrm{DTT}$ ), then $2.5 \mu \mathrm{L}$ of $4 \times$ compound solution and $5 \mu \mathrm{L}$ of $2 \times$ kinase solution were added to a 384-well plate (OptiPlate-384, PerkinElmer). The resulted solutions were briefly mixed, centrifuged and incubated for $5 \mathrm{~min}$. Finally, $2.5 \mu \mathrm{L}$ of $4 \times$ ATP/TK peptide (ATP at $K_{m}$ for each kinase $₫$ solution was added to the reaction system to initiate the reaction. The enzymatic reactions were carried out for 60-120 min at $23^{\circ} \mathrm{C}$, then terminated by adding $5 \mu \mathrm{L}$ of detection solution containing TK antibody-cryptate and 5 $\mu \mathrm{L}$ of Streptravidin-XL-665. The mixtures were incubated for additional 1 hour at $23^{\circ} \mathrm{C}$. The fluorescent signals were read using EnVision multilable plate reader (PerkinElmer). $\mathrm{IC}_{50}$ values of the compounds were generated using GraFit software (Version 6.0) and presented at the mean of three independent experiments performed in duplicate. The selective inhibition of SY-707 against 96 kinases was performed by Cerep.

Cell proliferation assay. MCF-7, T47D, MDA-MB-231, MIA Paca-2, PANC-1, SW620, HT-29, U87MG, and A549 cell lines were purchased from the cell bank of Chinese Academy of Science (Shanghai, China). Cells were cultured in DMEM or RPMI-1640 supplemented with $10 \%$ FBS, and $50 \mathrm{IU}$ penicillin/streptomycin in a humidified atmosphere with $5 \% \mathrm{CO}_{2}$ at $37{ }^{\circ} \mathrm{C}$. The effects of SY-707 on cell proliferation were measured using Promega's kits (CellTiter-Glo ${ }^{\circledR}$ or CellTiter-Blue ${ }^{\circledR}$ Cell Viability Assay). 
Cells were seeded in 96-well plate at low destiny with $195 \mu \mathrm{L}$ medium per well. The stock solutions of compounds in 100\% DMSO were 3-fold serially diluted from $10 \mathrm{mM}$ to $0.3 \mathrm{nM}$, and $4 \mu \mathrm{L}$ of solution at each concentration was transfered to $96 \mu \mathrm{L}$ of serum free medium (SFM), then $5 \mu \mathrm{L}$ of the resulted solution was added to each well. After treatment for 72 hours, 25 or $35 \mu \mathrm{L}$ of CellTiter-Glo ${ }^{\circledR}$ (for adherent cells) or CellTiter-Blue ${ }^{\circledR}$ (for suspension cells) reagent was added to each well, and the resulted mixture was further incubated at RT for 10 minutes or 4 hours. The luminescence/fluorescent signals were measured using EnVision ${ }^{\circledR}$ multi-labelled plate reader (PerkinElmer) or FlexStation 3 (Molecular Devices) and $\mathrm{IC}_{50}$ values were calculated using Prism ${ }^{\circledR}$ software (Version 5.0) and reported at the average of three independent experiments performed in duplicate.

Western blot. The cells were collected and lysed, and total protein concentrations were determined with a Bio-Rad BCA ${ }^{\circledR}$ kit. Equal amounts of cell lysates were loaded onto 10\% SDS gel and separated by electrophoresis. Separated proteins were then electro-transferred onto polyvinylidene fluoride (PVDF) membranes (Millipore, Bedford, MA). After being blocked with Tris-buffered saline (TBS) containing $0.1 \%$ Tween-20 and 5\% bovine serum albumin (BSA), the membranes were incubated with primary antibodies at room temperature for 2 hours or at $4{ }^{\circ} \mathrm{C}$, then washed with TBS containing $0.1 \%$ Tween-20 and followed by treatment with the horseradish peroxidase (HRP)-conjugated secondary antibody at room temperature for another 1 hour. The targeted proteins were visualized using an enhanced chemiluminescence (ECL) plus system (Thermo Fisher Scientific, Waltham, MA). Primary antibodies against phospho-FAK, phospho-ERK, phospho-AKT, FAK, AKT and $\beta$-Actin and the HRP conjugated goat anti-rabbit IgG were purchased from Cell Signaling Technology.

Flow cytometry. MCF-7 and T47D cells were treated with 0.1 \% DMSO (control) or with SY-707 solutions at indicated concentrations for 24 hours at $37^{\circ} \mathrm{C}$, then the cells were harvested by centrifugation and washed twice with PBS. Cell apoptosis was determined with a PE Annexin V Apoptosis Detection Kit (BD Biosciences, San Jose, CA) following the manufacture's guides. Breifly, cells were resuspended with 100 $\mu \mathrm{L}$ of binding buffer containing with Annexin $\mathrm{V}$ and 7-AAD. After 15 minutes incubation at room tempreture in the dark, $400 \mu \mathrm{L}$ of binding buffer was added to the mixture and the cells were analyzed with a Guava flow cytometer (Millipore, Bedford, MA). Cell cycle arrest was evaluated by incorporation of propidium (PI) (Sigma-Aldrich, St.Louis, MO) into the permeablized cells. After being treated with DMSO or compounds for 24 hours, cells were harvested, washed twice with cold PBS, fixed with ice-cold $70 \%$ ethanol followed by a secondary staining step with staining buffer $(0.25 \mathrm{mg} / \mathrm{mL}$ Rnase, $0.025 \mathrm{mg} / \mathrm{mL} \mathrm{PI}$, in PBS) for 1 hour at $37^{\circ} \mathrm{C}$. Compound regulation of cell-cycle was analyzed using a Guava flow cytometer (Millipore, Bedford, MA). Data were analyzed with FlowJo software.

Would healing assay. Cells were plated in 6-well plate, cell monolayer (with 70-80\% confluence in plate) was gently and slowly scratched with a new $1 \mathrm{ml}$ pipette tip across the center of the well. After scratching, the wells were gently washed with PBS twice to remove the detached cells, and replenished with fresh medium containing indicated concentration of compounds. The treated cells grew for additional 24 hours 
then graphed with a microscope, and the migration distance were calculated and analysized to evaluate effects of compounds.

Tumor xenograft studies in nude mice. Female athymic nude mice (5-6 week) were used for all the in vivo studies. $5 \times 10^{6}$ or $5 \times 10^{5}$ of T47D or $4 \mathrm{~T} 1$ in $100 \mu \mathrm{L}$ of serum-free medium were planted subcutaneously on the right and left flanks of mice. Tumor volumes were monitored by caliper measurement using the formula: tumor volume $\left(\mathrm{mm}^{3}\right)=\left(\mathrm{w}^{2} \mathrm{xl}\right) / 2$, where $\mathrm{w}=$ width and $\mathrm{I}=$ length. When the tumor volumes reached 150 200 $\mathrm{mm}^{3}$, mice were randomized to groups (8-10 mice per group) and treated with compounds or vehicle by oral gavage every day for 2-4 weeks. The tumor volumes and body weight were measured every 2-3 days. Tumor growth inhibition (TGI) and body weight changes were calculated compared to the control groups. Statistical significance of the treatment groups compared to vehicle group was evaluated with t-test. All the experiments were carried out in accordance with the guidelines of institutional animal care and use committee (IACUC) of Shouyao Holdings and approved by IACUC of Shouyao Holdings.

Anti-metastasis studies in nude mice. Establishment of mouse 4T1 xenograft model and compound administration was performed as described previously. Metastasis was detected in mouse lungs after the mice were sacrificed at the last dosing day. The lungs of each mouse were excised immediately and fixed with $4 \%$ of paraformaldehyde for 24 hours, then the lungs were photograph and the metastatic nodules in lungs were manually counted and the data was analyzed for compound evaluation.

Pharmacokinetic studies. Female SD rats (5-6 week) or beagle dogs were administrated with SY-707 at a single dose of $5 \mathrm{mg} / \mathrm{kg}$ and blood samples were taken sequentially from 2 minutes to 24 hours after compound administration. The serum samples were collected by centrifugation and stored at $-80^{\circ} \mathrm{C}$. Compound concentrations in the sample serums were further analyzed by LC-MS/MS (API-4000, Applied Biosystem Inc.), and pharmacokinetic parameters $\left(T_{1 / 2}, T_{\text {max }}, C_{\text {max }}, A U C_{0-1 N F}\right)$ were generated using WinNonlin software (WinNonlin Professional, version 6.2).

Statistical analysis. Two-tailed $t$ tests were performed. $\mathrm{P}<0.05$ was regarded to be statistically significant. The data are presented as the mean \pm standard deviation (SD).

\section{Results}

SY-707 is a multi-kinase inhibitor against ALK, FAK and IFG1R. SY-707 was developed as an ALK inhibitor primitively. It showed very potent inhibition on ALK kinase with an $\mathrm{IC}_{50}$ value of $2.4 \mathrm{nM}$ (Fig.1B). To evaluate in vitro enzymatic potency and selectivity of SY-707, a kinase panel screening on 96 kinases was performed to determine inhibitory activities of SY-707 at concentration of $1 \mathrm{mM}$. Besides ALK, SY707 was able to inhibit several other kinases, such as FAK, Pyk2, LTK, IRK and IGF1R markedly with IC 50 values around 1-10 nM (Fig.1B). Then, enzymatic kinetic analysis on ALK and FAK was executed to explore mechanisms of inhibition of SY-707. Activities of ALK or FAK were estimated at various concentration of ATP in presence of SY-707 or not. SY-707-mediated inhibition on ALK kinase activity can 
be significantly attenuated with increasing concentration of ATP (Fig.1C). SY-707 had higher affinity to free ALK enzyme with a dissociation constant $\left(K_{i}\right)$ value of $0.90 \mathrm{nM}$ than the enzyme-ATP complex with a $K_{i}^{\prime}$ value of $19.2 \mathrm{nM}$. Lineweaver-Burk plots (Double-reciprocal plots of $1 / v$ versus $1 /[s]$ ) (inset of Fig. $1 C$ ) illustrated that the four plots were intersected on $Y$ axes, indicating that SY-707 inhibited ALK kinase as an ATP-competitive inhibitor. Similarly, SY-707 also preferred to combine free FAK enzyme with a dissociation constant $\left(K_{i}\right)$ value of $0.56 \mathrm{nM}$ than to combine the enzyme-ATP complex with a $K_{i}^{\prime}$ value of $16.1 \mathrm{nM}$. And the four Double-reciprocal plots were also intersected on $\mathrm{Y}$ axes, suggesting an ATPcompetitive inhibitory behavior of SY-707 for FAK kinase (inset of Fig.1D). Since there was sequence similarity between catalytic domains of multiple kinases, it was reasonable that SY-707 depressed multiple kinases in an ATP-dependent manner.

SY-707 inhibits proliferation of breast cancer cell lines. To evaluate the cellular anti-tumor activities of SY707, the cell proliferation studies were executed to evaluate the inhibitory effects of SY-707 on a series of cell lines including breast cancer (MCF-7, MDA-MB-231, T47D), pancreas cancer (MIA Paca-2, PANC-1), glioblastoma (U87MG), colon cancer (SW620), melanoma carcinoma (HT29), and lung cancer (A549). SY-707 significantly suppressed the growth of the most of solid cancer cells with $\mathrm{IC}_{50}$ values of $0.5-10$ $\mu \mathrm{m}$ (Fig 2A). Late apoptosis induced by SY-707 was observed in MDA-MB-231 cells dose-dependently on concentration of SY-707 (Fig.2B). The cleaved poly-ADP ribose polymerase (PARP) was markedly detected in these treated cells and the amounts of cleaved PARP were correlated with the concentration of compound, meaning that SY-707 induced cell apoptosis by the way of formation of active apoptotic executor PARP (Fig.2C). Meanwhile, SY-707 was able to induce pronounced G2/M phase arrest and a decrease in the percentage of cells in S phase in a dose-dependent manner (Fig. 2D).

SY-707 suppresses FAK signaling pathway in breast cancer. To investigate the underlying mechanisms of SY-707 inhibition on cell proliferation, we evaluated the effects of SY-707 on FAK signaling pathways. SY707 dose-dependently suppressed the phosphorylation of FAK at Tyrosine397 and the phosphorylation of downstream protein ERK in MDA-MB-231 and T47D cells (Fig. 3A, B). More importantly, SY-707 displayed higher potency than reference compounds PF-562271 on the inhibition of phosphorylation ERK.

SY-707 blocked cell migration and adhesion of breast cancer cells. As FAK played key roles in cancer migration and metastasis, the effects of SY-707 on cell invasion and cell adhesion was evaluated. It was found that migration distances were significantly decreased in SY-707 treatment group compared to that in control group in a dose-dependently manner. Meanwhile, the significant reduction of cell migration was also observed in PF-562271 treated group (Fig.4A). In another hand, SY-707 characteristically repressed cell adhesion in a dose-dependent manner (Fig.4B). Thus, SY-707 suppressed cell invasion and cell adhesion, and this also supplied evidences for further exploration of anti-metastasis potency in in vivo studies.

SY-707 has anti-tumor activity in vivo via FAK inhibition. Since SY-707 showed excellent in vitro potency in enzymatic and cellular assays, we further evaluated its pharmacokinetics profile in SD rats and beagle dogs by oral (peros, PO) before in vivo efficacy studies. SY-707 was eliminated slowly with a $t_{1 / 2}$ of 12.1 
and 9.72 hours in SD rat or beagle dog respectively, displayed high exposure in animal plasma with mean $\mathrm{AUC}_{0-\mathrm{INF}}$ of $10964 \mathrm{hr} \mathrm{ng} / \mathrm{mL}$ for rat and $459 \mathrm{hr}$ ng/mL for beagle dog correspondingly (Table. 1). In addition, SY-707 also had appropriate clearance parameters and oral bioavailability in these two species.

Table.1 Pharmacokinetics parameters of SY-707

\begin{tabular}{|c|c|c|c|c|c|c|}
\hline \multirow[t]{2}{*}{ Species } & \multicolumn{3}{|c|}{ Rat 5 mg/kg (PO) } & \multicolumn{3}{|c|}{ Beagle Dog $5 \mathrm{mg} / \mathrm{kg}$ (PO) } \\
\hline & Male & Female & All & Male & Femal & $\overline{\text { All }}$ \\
\hline$t_{1 / 2}(\mathrm{hr})$ & 13 & 11.2 & 12.1 & 9.33 & 10.1 & 9.72 \\
\hline $\mathrm{T}_{\max }(\mathrm{hr})$ & 3.33 & 4.33 & 3.83 & 3 & 2.67 & 2.83 \\
\hline $\mathrm{C}_{\max }(\mathrm{ng} / \mathrm{mL})$ & 909 & 639 & 774 & 37.8 & 34.2 & 36 \\
\hline $\mathrm{AUC}_{0-\mathrm{INF}}\left(\mathrm{hr}{ }^{*} \mathrm{ng} / \mathrm{mL}\right)$ & 13335 & 8593 & 10964 & 479 & 438 & 459 \\
\hline Cl_F_obs $(\mathrm{mL} / \mathrm{hr} / \mathrm{kg})$ & 378 & 586 & 482 & 11257 & 12131 & 11694 \\
\hline $\mathrm{MRT}_{\text {INF_obs }}(\mathrm{hr})$ & 15.5 & 13.8 & 14.7 & 13.6 & 13.7 & 13.6 \\
\hline $\mathrm{F}(\%)$ & 38.3 & 33.2 & 36.2 & 23.8 & 18.8 & 21.1 \\
\hline
\end{tabular}

Due to strong cell growth inhibition potency of SY-707, we firstly evaluate its in vivo anti-cancer activities in mouse xenograft model bearing T47D cells. In this model, mice were orally administered with vehicle or SY-707 at dose of 100,50 or $25 \mathrm{mg} / \mathrm{kg}$ twice a day (BID) after tumor size reached to $150-300 \mathrm{~mm}^{3}$. The results showed that SY-707 suppressed T47D tumor growth in a dose-dependent manner (Fig. 5A). After treatment with SY-707 for 21 days, tumor growth inhibition was estimated at $61.0 \%(p<0.01), 39 \%(p<$ $0.05), 31 \%(p<0.05)$ in 100,50 , and $25 \mathrm{mg} / \mathrm{kg}$ administrational groups respectively, while only reached $20 \%$ in reference compound PF-562217 dosing group.

To explore the relationship between the in vivo anti-tumor growth activities and the inhibition on FAK signaling transduction, pharmacokinetics / pharmacodynamics studies were performed in T47D xenograft model. Phosphorylation level of FAK at Tyrosine397 in SY-707 treatment groups were significantly decreased (Fig. 5B). Meanwhile, higher concentration of SY-707 in tumor samples were also detected compared to that in plasma, suggesting that SY-707 was able to access to tumor tissue with high exposure.

Next step, we evaluated the combination effects of SY-707 and paclitaxel (an approved drug for breast cancer therapy) in T47D xenograft model. Mice bearing T47D breast cancer were divided randomly into four groups and received vehicle, SY-707 (100 mg/kg, once a day (QD)), paclitaxel (10 mg/kg, once a week (QW)) or SY-707 plus paclitaxel respectively. After dosing for 24 days, tumor growth inhibition of xenograft mice in SY-707 (100 mg/kg, QD) and paclitaxel (10 mg/kg, QW) treatment groups was $14 \%$ and $24 \%(p<0.05)$ respectively, while that in combinational group achieved to $66 \%(p<0.01)$ (Fig. $5 \mathrm{C})$. 
In 4T1 xenograft model, SY-707 was administered with or without paclitaxel at indicated concentration, and then anti-tumor activities were evaluated three times a week. Only slight suppression on 4T1 tumor growth was observed in all of monotherapy groups, while significant inhibition on tumor growth was detected in combined group ( $100 \mathrm{mg} / \mathrm{kg}$ SY-707 plus $20 \mathrm{mg} / \mathrm{kg}$ paclitaxel) with a tumor growth inhibition value of $51.5 \%(p<0.05)$ (Fig. 5D).

SY-707 repressed spontaneous metastasis of breast cancer to the lung. Cancer metastasis from primary area to other organs is a major cause of morbidity and mortality for breast cancer, since SY-707 displayed notable anti-migration activities in cellular assays, in vivo anti-metastasis effects were consequently evaluated in 4T1 xenograft models, in which the tumor metastasized to the lung spontaneously[17]. Alone treatment of SY-707 (100 mg/kg, PO, QD), paclitaxel, or paclitaxel (HS-15) (20 $\mathrm{mg} / \mathrm{kg}$ or $10 \mathrm{mg} / \mathrm{kg}$, IV (intravenous injection), QW) reduced the number of metastatic nodules in lungs but not statistically significantly (Fig. 6C, D, G, H). In contrast, combination of SY-707 and paclitaxel was able to eliminate the number of metastatic nodules in nude mice bearing $4 \mathrm{~T} 1$ breast cancer significantly. Metastatic nodules in $100 \mathrm{mg} / \mathrm{kg}$ SY-707 (PO, QD) plus $20 \mathrm{mg} / \mathrm{kg}$ paclitaxel (IV, QW) group decreased for more than $40 \%$ compared to that in vehicle group $(p<0.01)$, and the number in $100 \mathrm{mg} / \mathrm{kg} \mathrm{SY}-707$ (PO, QD) plus $10 \mathrm{mg} / \mathrm{kg}$ paclitaxel (IV, QW) group also decreased for $31.1 \%$ compared to vehicle group $(p<$ 0.05) (Fig. 6E, F). In conclusion, SY-707 plus paclitaxel notably depressed cancer metastasis to lungs from primary area.

\section{Discussions}

Development of kinase inhibitors in drug discovery was an efficacious approach for treatment of multiple cancers and benefitted to thousands of cancer patients in the past few decades[18-20], however, cancer metastasis made most of the therapies failed possibly because of the spread, invasion and relocation of metastatic cancer cells from primary areas to other organs in patients. Therefore, discovery of antimetastasis drug became an attractive way to improve current therapy and to expand pipelines for many pharmaceutical companies.

SY-707 is evaluated in Phase II clinical trial for the treatment of ALK positive non-small cell lung cancer. In this study, the results showed that it behaved as an ATP-competitive inhibitor against multiple kinases such as ALK, FAK, Pyk2 and IGF1R and others. Thus, we systematically explored its inhibitory potency on cancer growth and cancer metastasis via suppressing FAK in breast cancer to explore additional indications for future clinical studies. SY-707 strongly suppressed the tumor cell growth, which was well associated with the dose-dependent induction of cell apoptosis and cell cycle arrest at G2/M phase (Fig2). PARP is a key DNA repair factor. Its inhibitors have been tested in BRAC1/2-mutant tumors and are in ongoing trials for the treatment of various malignancies including breast cancer. Cleaved PARP is released from apoptotic cells and as a biomarker for the apoptotic response of tumor cells[21]. SY-707 promoted the formation of cleaved PARP (Fig2C). Overall, the results indicated that SY-707 potently attenuated cell growth via blocking cell cycle progression and inducing active PARP to execute apoptosis in breast cancer cells. 
FAK was a key regulator of cell invasion, cell migration and cell proliferation of breast cancer[22]. Since SY-707 markedly inhibited FAK in kinase assays (Fig1B), we consequently explore the effects of SY-707 on FAK signaling cascades, and it was found that SY-707 depressed FAK signaling pathways via decreasing its phosphorylation and its downstream proteins (Fig3). More importantly, SY-707 was able to attenuated invasion and migration (Fig4), indicating that SY-707 affected the biological functions via inhibiting FAK in cancer cells.

Further pharmacokinetic studies demonstrated that SY-707 owned druggable pharmacokinetics parameters in SD rat and beagle dog, such as slow metabolization (long $t_{1 / 2}$ of 12.1 hours in rat and 9.72 hours in beagle dog) and high oral exposures in plasma (AUC of $10964 \mathrm{hr} n \mathrm{ng} / \mathrm{mL}$ in rat) (Table.1). SY707 displayed significant suppression on tumor growth of T47D or 4T1 breast cancer in animal models, and it had significantly synergistic effects combined with paclitaxel. The inhibition of FAK phosphorylation in T47D tumors by SY-707 was well correlated with its concentrations in tumor tissues, indicating a mechanism-based blockage of in vivo tumor growth by it (Fig5B).

Besides tumor growth inhibition, attenuation of cancer metastasis from primary region to the lungs was also observed in animal models in SY-707 plus paclitaxel groups. It was consistent with its cellular inhibitory potency on cancer cell invasion and attachment, meaning that SY-707 depressed cancer metastasis possibly by the way of inhibiting FAK pathways. Based on the current results, treatment with SY-707 possibly provided an approach of blocking cancer metastasis in the future therapy for breast cancer. In particular, SY-707 had better pharmacokinetics parameters than known FAK and ALK inhibitor PF-562271, thus it should behaved better efficacy in clinical studies. Collectively, SY-707 could be developed as a multiple drug candidate for multiple indications including breast cancer besides ALK positive NSCLC.

\section{Conclusion}

SY-707 was a multiple kinase inhibitor against several kinases like FAK, IGF1R, ALK, Pyk2 and others. It not only inhibited growth of breast cancer, but also eliminated invasion of cancer in vitro and in vivo, thus supplying a therapeutically potential for treatment of metastatic breast cancer and expanding additional indications in future clinical studies.

\section{Abbreviations}

FAK, Focal adhesion kinase; NSCLC, non-small cell lung cancer; TGI, tumor growth inhibition; QD, once a day; QW, once a week; PO, peros; IV, intravenous injection

\section{Declarations}

\section{Ethics approval and consent to participate}


This study was approved by the institutional animal care and use committee (IACUC) of Shouyao Holdings and approved by IACUC of Shouyao Holdings.

\section{Consent for publication}

All authors agreed on the manuscript.

\section{Availability of data and materials}

The data supporting the conclusions of this paper are included within the manuscript.

\section{Competing interests}

The authors declare that there is no conflict of interest regarding the publication of this paper

\section{Funding}

This work was supported by the National Major Scientific and Technological Special Project for "Significant New Drugs Development" during the Twelfth Five-year Plan Periods (Foundation No. 2012ZX09401007 and 2015ZX09101001).

\section{Contributions}

$\mathrm{BL}$ and QC conceived and designed the experiments. PL, YS, JN and XL performed the experiments. $\mathrm{BL}$ and QC analyzed the data. PL and QC wrote the manuscript. All authors read and approved the manuscript.

\section{Acknowledgements}

None.

\section{References}

1. Siegel RL, Miller KD, Jemal A: Cancer statistics, 2019. CA: a cancer journal for clinicians 2019, 69(1):7-34.

2. Weigelt B, Peterse JL, van 't Veer LJ: Breast cancer metastasis: markers and models. Nature reviews Cancer 2005, 5(8):591-602.

3. Tong CWS, Wu M, Cho WCS, To KKW: Recent Advances in the Treatment of Breast Cancer. Frontiers in oncology 2018, 8:227.

4. Yoon H, Dehart JP, Murphy JM, Lim ST: Understanding the roles of FAK in cancer: inhibitors, genetic models, and new insights. The journal of histochemistry and cytochemistry : official journal of the Histochemistry Society 2015, 63(2):114-128. 
5. Golubovskaya VM, Cance WG: FAK and p53 protein interactions. Anti-cancer agents in medicinal chemistry 2011, 11(7):617-619.

6. Shtam T, Naryzhny S, Samsonov R, Karasik D, Mizgirev I, Kopylov A, Petrenko E, Zabrodskaya Y, Kamyshinsky R, Nikitin D et al: Plasma exosomes stimulate breast cancer metastasis through surface interactions and activation of FAK signaling. Breast cancer research and treatment 2019, 174(1):129-141.

7. Tancioni I, Miller NL, Uryu S, Lawson C, Jean C, Chen XL, Kleinschmidt EG, Schlaepfer DD: FAK activity protects nucleostemin in facilitating breast cancer spheroid and tumor growth. Breast cancer research : $B C R 2015,17: 47$.

8. Golubovskaya VM, Ylagan L, Miller A, Hughes M, Wilson J, Wang D, Brese E, Bshara W, Edge S, Morrison $C$ et al: High focal adhesion kinase expression in breast carcinoma is associated with lymphovascular invasion and triple-negative phenotype. BMC cancer 2014, 14:769.

9. Lee BY, Timpson P, Horvath LG, Daly RJ: FAK signaling in human cancer as a target for therapeutics. Pharmacology \& therapeutics 2015, 146:132-149.

10. Lv PC, Jiang AQ, Zhang WM, Zhu HL: FAK inhibitors in Cancer, a patent review. Expert opinion on therapeutic patents 2018, 28(2):139-145.

11. Sulzmaier FJ, Jean C, Schlaepfer DD: FAK in cancer: mechanistic findings and clinical applications. Nature reviews Cancer 2014, 14(9):598-610.

12. McLean GW, Carragher NO, Avizienyte E, Evans J, Brunton VG, Frame MC: The role of focaladhesion kinase in cancer - a new therapeutic opportunity. Nature reviews Cancer 2005, 5(7):505-515.

13. Stokes JB, Adair SJ, Slack-Davis JK, Walters DM, Tilghman RW, Hershey ED, Lowrey B, Thomas KS, Bouton AH, Hwang RF et al: Inhibition of focal adhesion kinase by PF-562,271 inhibits the growth and metastasis of pancreatic cancer concomitant with altering the tumor microenvironment. Molecular cancer therapeutics 2011, 10(11):2135-2145.

14. Roberts WG, Ung E, Whalen P, Cooper B, Hulford C, Autry C, Richter D, Emerson E, Lin J, Kath J et al: Antitumor activity and pharmacology of a selective focal adhesion kinase inhibitor, PF-562,271. Cancer research 2008, 68(6):1935-1944.

15. Green AR, Caracappa D, Benhasouna AA, Alshareeda A, Nolan CC, Macmillan RD, Madhusudan S, Ellis IO, Rakha EA: Biological and clinical significance of PARP1 protein expression in breast cancer. Breast cancer research and treatment 2015, 149(2):353-362.

16. Schultze A, Fiedler W: Therapeutic potential and limitations of new FAK inhibitors in the treatment of cancer. Expert opinion on investigational drugs 2010, 19(6):777-788. 
17. Milas L, Peters LJ, Ito H: Spontaneous metastasis: random or selective? Clinical \& experimental metastasis 1983, 1(4):309-315.

18. Fabbro D, Ruetz S, Buchdunger E, Cowan-Jacob SW, Fendrich G, Liebetanz J, Mestan J, O'Reilly T, Traxler P, Chaudhuri B et al: Protein kinases as targets for anticancer agents: from inhibitors to useful drugs. Pharmacology \& therapeutics 2002, 93(2-3):79-98.

19. Zwick E, Bange J, Ullrich A: Receptor tyrosine kinases as targets for anticancer drugs. Trends in molecular medicine 2002, 8(1):17-23.

20. Bennasroune A, Gardin A, Aunis D, Cremel G, Hubert P: Tyrosine kinase receptors as attractive targets of cancer therapy. Critical reviews in oncology/hematology 2004, 50(1):23-38.

21. Liu CY, Chu PY, Huang CT, Chen JL, Yang HP, Wang WL, Lau KY, Lee CH, Lan TY, Huang TT et al: Varlitinib Downregulates HER/ERK Signaling and Induces Apoptosis in Triple Negative Breast Cancer Cells. Cancers 2019, 11(1).

22. Luo M, Guan JL: Focal adhesion kinase: a prominent determinant in breast cancer initiation, progression and metastasis. Cancer letters 2010, 289(2):127-139.

\section{Figures}

A

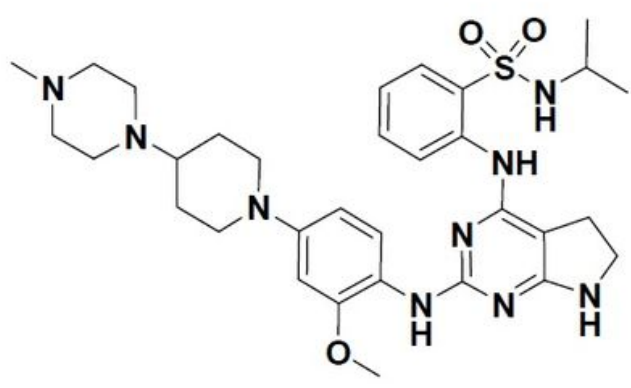

$\mathrm{C}$

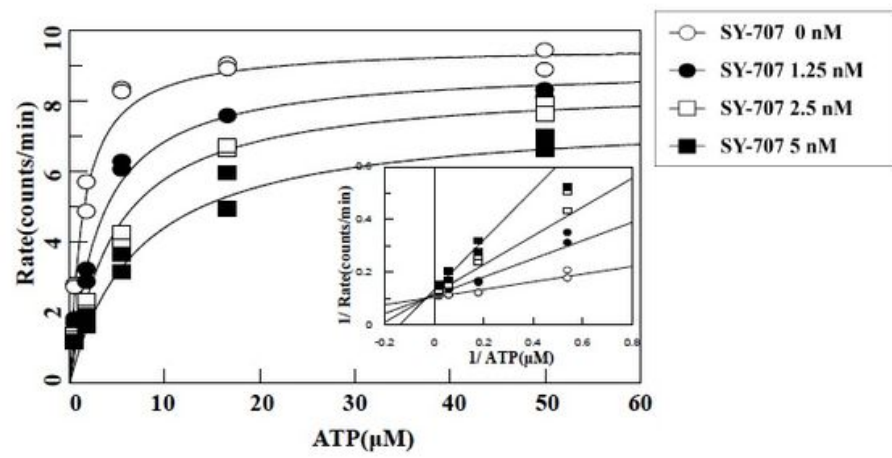

B

\begin{tabular}{cc}
\hline Enzyme & IC $_{50}(\mathrm{nM})$ \\
\hline FAk & $1.6 \pm 0.5$ \\
Pyk2 & $2.4 \pm 0.8$ \\
ALK & $2.4 \pm 0.7$ \\
LTK & $1.9 \pm 0.18$ \\
IRK & $7.9 \pm 0.30$ \\
IGF1R & $10.6 \pm 0.58$ \\
Ack & $28.7 \pm 3.5$ \\
Fer & $52.3 \pm 2.3$ \\
Fes & $81.8 \pm 19$ \\
\hline
\end{tabular}

$\mathrm{D}$

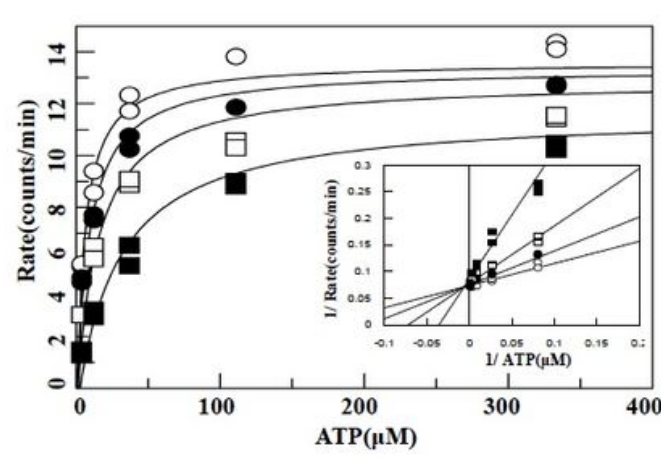

\section{Figure 1}


In vitro enzymatic inhibition of SY-707 against multiple kinases. (A) Chemical structure of SY-707. (B) Kinase inhibition profile of SY-707. (C) ALK enzyme activities were evaluated at various concentrations of ATP $(0-50 \mu \mathrm{M})$ in the presence of SY-707 (5 (○), $2.5(\bullet), 1.25(\square)$ and $0(\square)$ nM respectively). (D) FAK enzyme activities were evaluated at various concentrations of ATP $(0-333 \mu \mathrm{M})$ in the presence of SY-707 (2.7 (๑), $0.9(\bullet), 0.3(\square)$ and $0(\square)$ nM respectively).

A

\begin{tabular}{ccc}
\hline Cell line & Cancer Type & $\mathrm{IC}_{50}(\mu \mathrm{M})$ \\
\hline MCF-7 & breast & 0.59 \\
MDA-MB-231 & breast & 1.9 \\
T47D & breast & 1.8 \\
MIA PACA-2 & pancreas & 1.3 \\
PANC-1 & pancreas & 1.2 \\
SW620 & colon & 0.81 \\
HT29 & melonoma & 0.6 \\
U87MG & glioblastoma & $>10$ \\
A549 & lung & 5.7 \\
\hline
\end{tabular}

$\mathrm{C}$

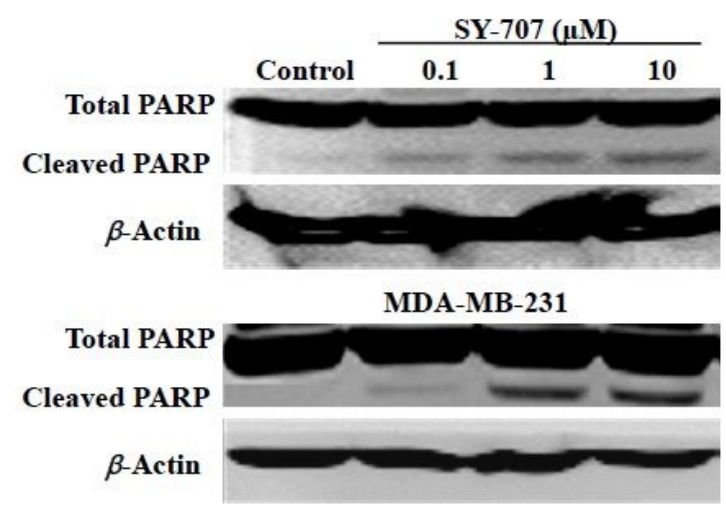

B

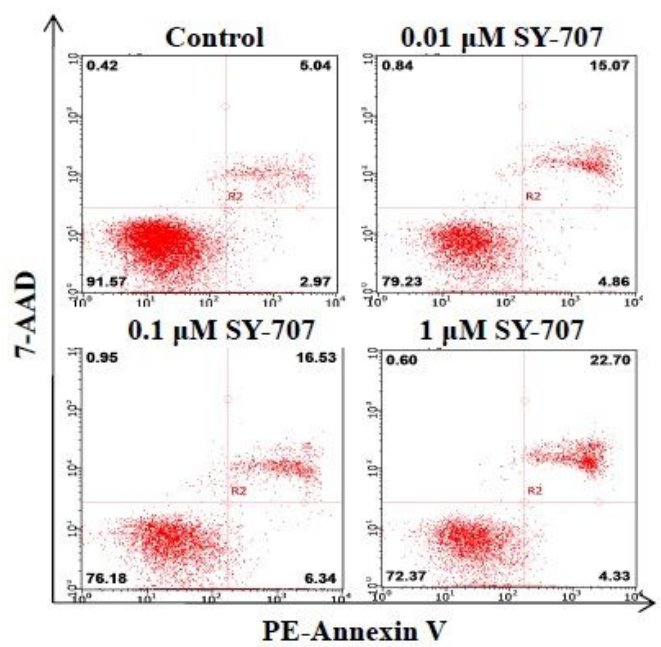

$\mathrm{D}$

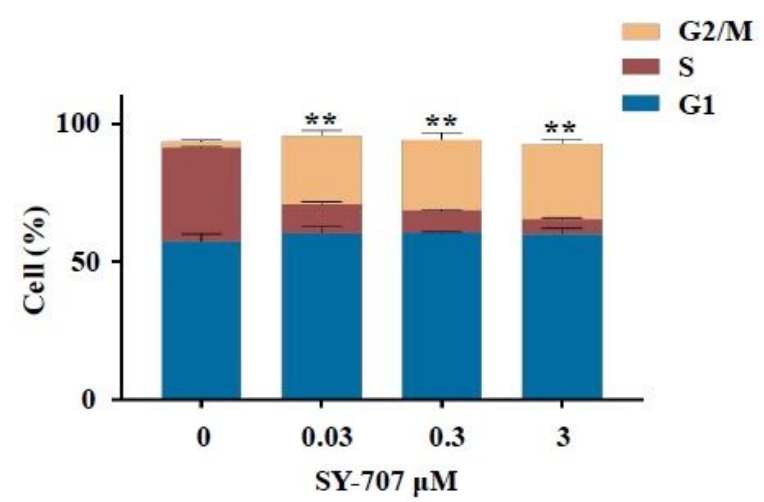

Figure 2

Effects of SY-707 on cell growth, cell apoptosis and cell cycle. (A) Anti-proliferation activities of SY-707 in a series of tumor cells. (B) Apoptosis of MDA-MB-231 cells treated with SY-707. Cells were cultured and treated with SY-707 under the indicated concentrations $(0.01,0.1$ and $1 \mu \mathrm{M}$ respectively) for 24 hours, then stained with Annexin-V and 7-AAD for cell apoptosis analysis with Guava flow cytometry. (C) T47D (upper) or MDA-MB-231 (down) cells were incubate with various concentration of SY-707 for 24 hours, then the cells were collected for Western blot with anti-PARP antibody. (D) Cell cycle of MDA-MB-231 cells treated with SY-707. Cells were harvested and washed with PBS for three times after incubation with the specified concentration of SY-707 for 24 hours, and cells were then labeled with propidium (PI) after fixing with ice ethanol. Cell cycle arrest was determined with Guava Flow cytometry. ${ }^{\star}, p<0.01$. 
A

MDA-MB-231

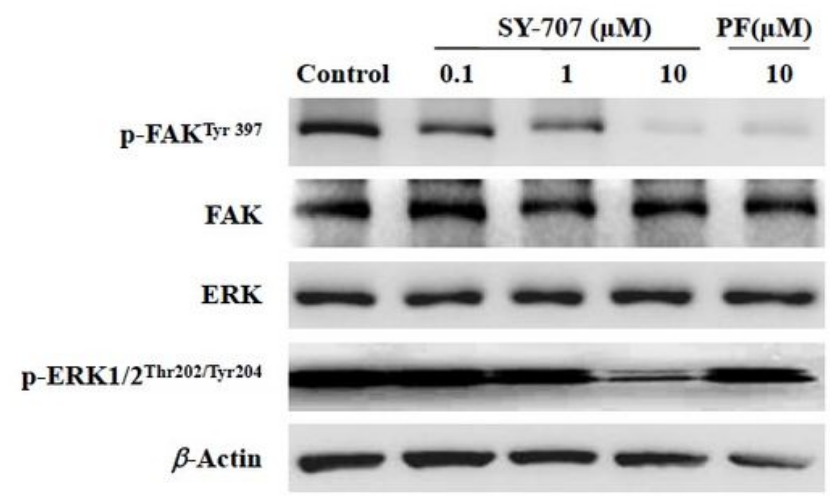

B

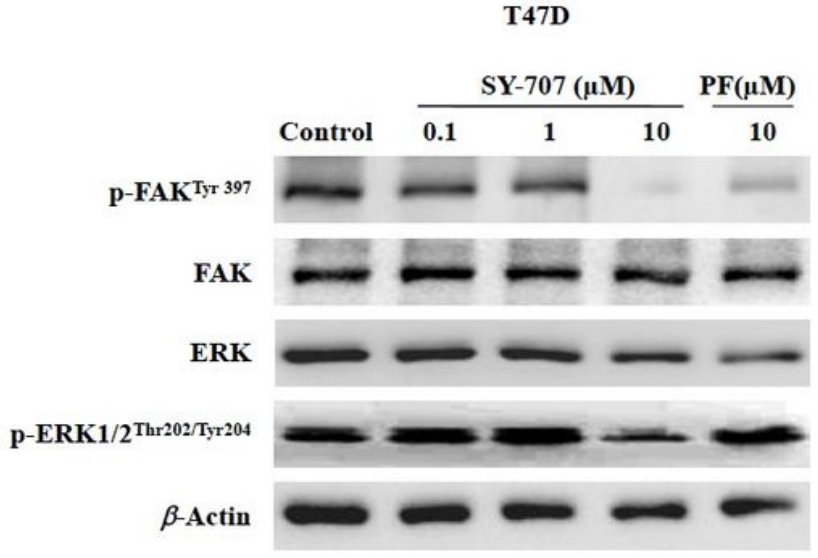

\section{Figure 3}

Inhibitory effects of SY-707 on the phosphorylation of FAK. Cells were treated with the indicated concentrations of SY-707 or PF-562271(PF) for 1 hour. Control cells received the drug vehicle with $0.1 \%$ DMSO. Cell lysates were analyzed by Western blot with specific antibodies. 
A

MDA-MB-231

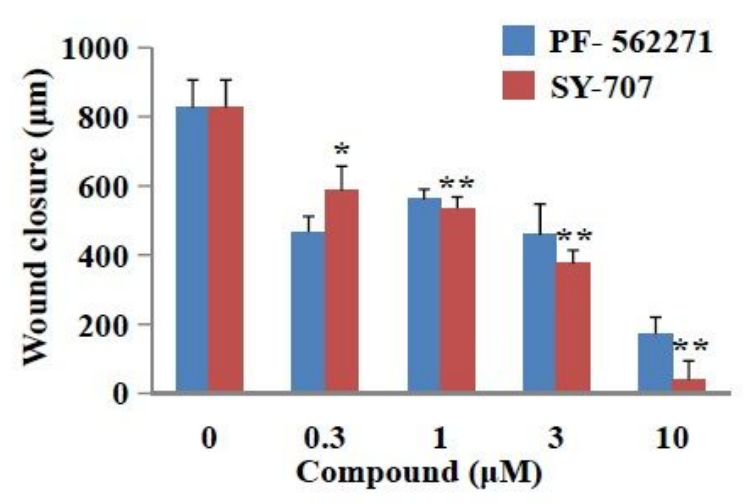

B

MDA-MB-231

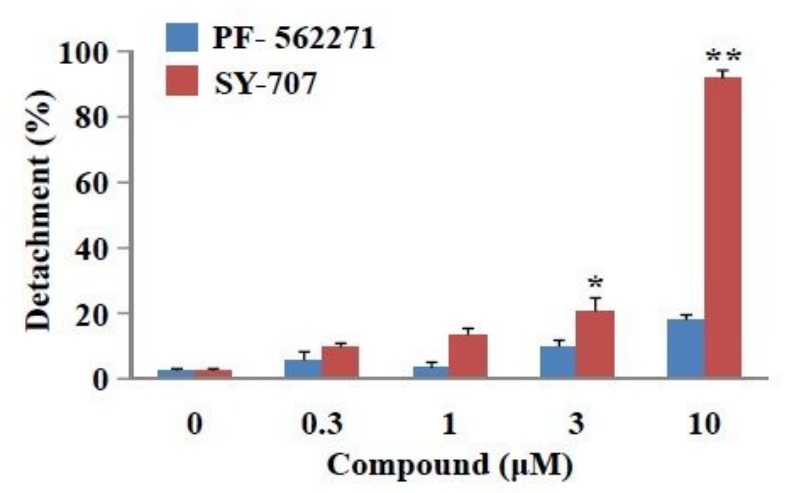

T47D

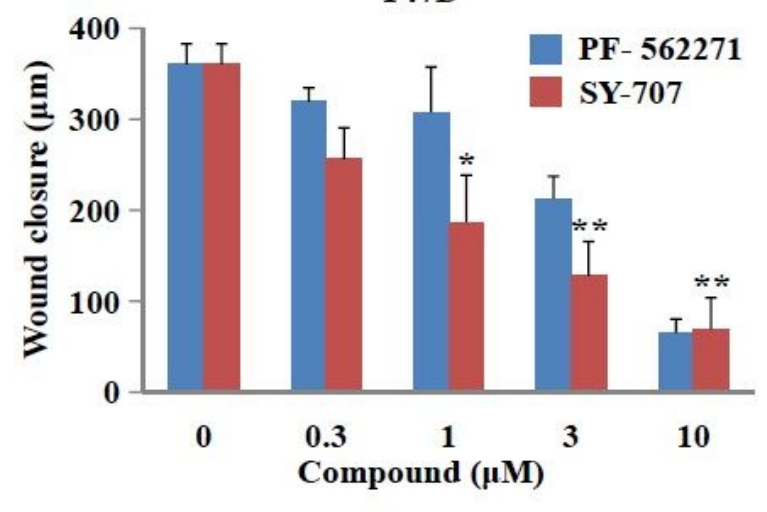

T47D

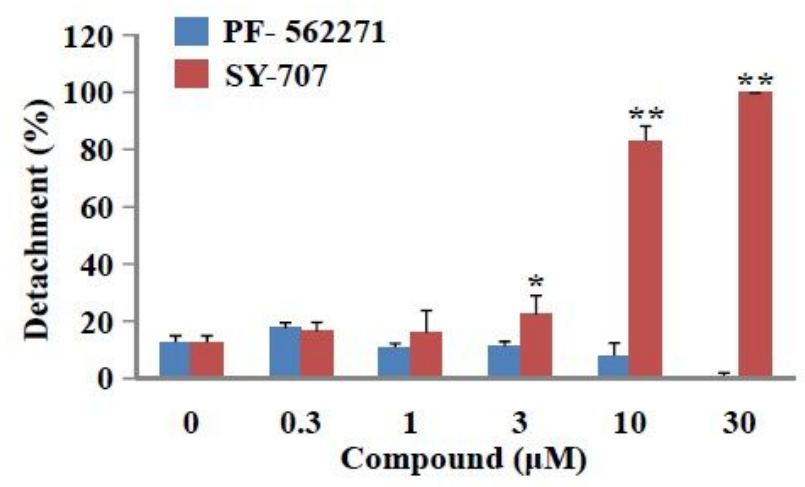

\section{Figure 4}

Block of SY-707 on cell migration and adhesion. (A) The migration distances of cell were calculated after the cells were incubated with SY-707 for 24 hours. (B) Effects of SY-707 on cell adhesion in MDA-MB-231 and T47D cells. The cells were plated on 6-well plates mixed with SY-707, and then the unattached cell numbers were counted after washing out. *, $p<0.05 ; * \star, p<0.01$. 
A

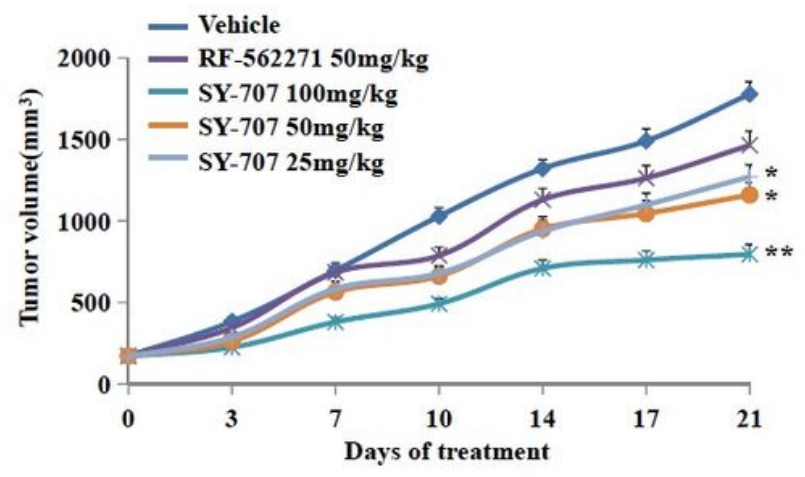

$\mathrm{C}$

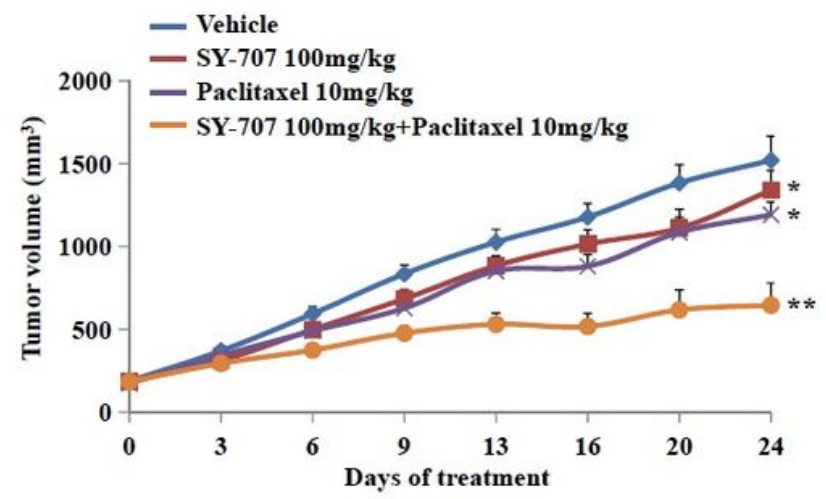

B

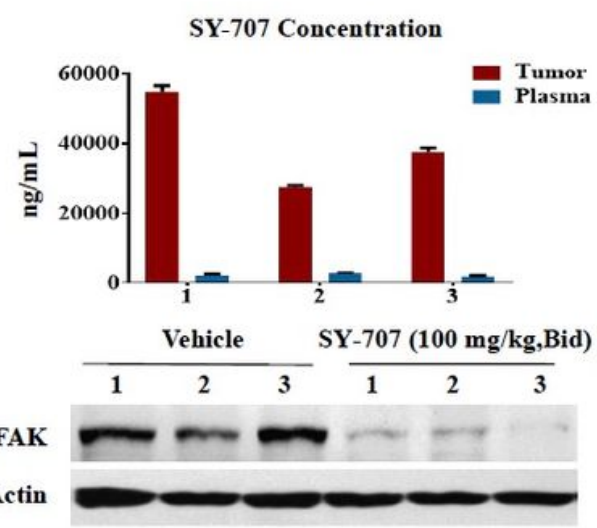

$\mathrm{D}$

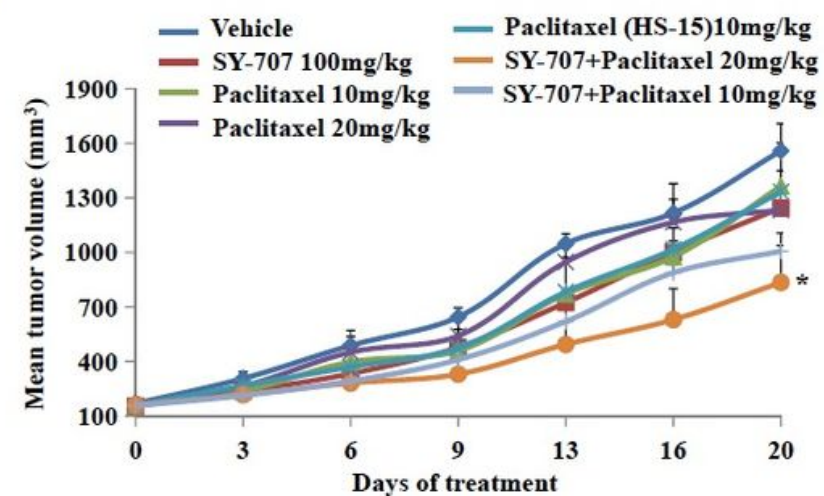

\section{Figure 5}

Anti-tumor activities of SY-707 in breast cancer xenografts. Xenograft tumors were established by inoculating T47D (A, B, C) cells or 4T1 (D) cells into the nude mice. Oral administration of SY-707 alone or combined with paclitaxel was initiated when tumors reached to an average of approximately 150-300 mm3 in volume and continued through the experiment. Tumor volume was measured on the indicated days with the mean tumor volume \pm SE indicated for each group (10 mice/group). Each treatment groups was compared to vehicle group with t-test. *, $p<0.05 ;{ }^{*}, p<0.01$. (B) The tumor tissues were harvested at 2 hours after the last dosage of SY-707, compound exposures in tumor tissue or plasma and the phosphorylation levels of FAK were analyzed. 


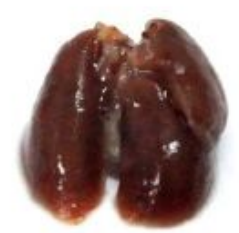

A

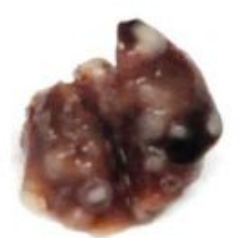

D

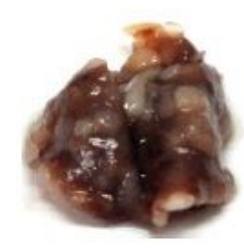

B

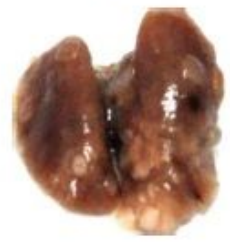

E

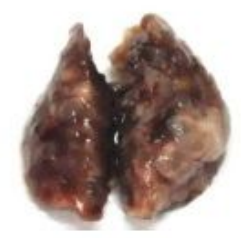

C

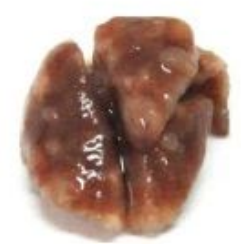

$\mathrm{F}$

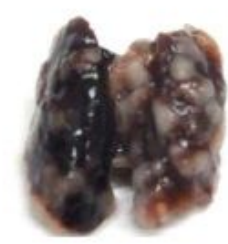

G

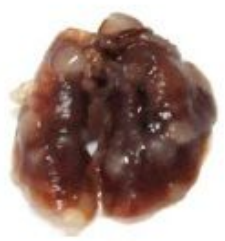

$\mathrm{H}$

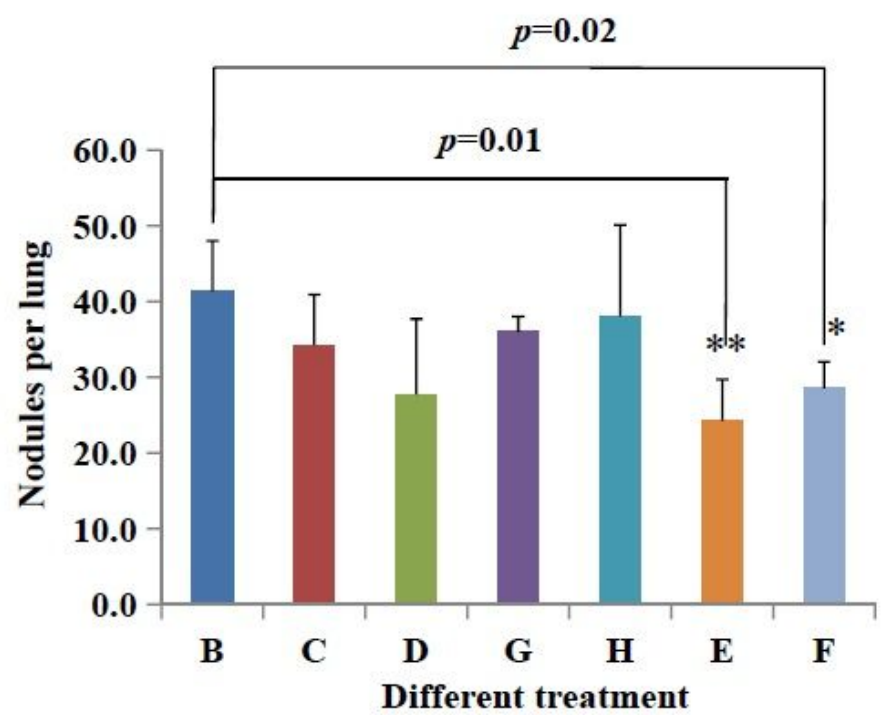

A: Normal mice

B: Vehicle (20\% SBE)

C: SY-707 100mg/kg

D: Paclitaxel 20mg/kg

E: SY-707 100mg/kg \& Paclitaxel 20mg/kg

F: SY-707 100mg/kg \& Paclitaxel 10mg/kg

G: Paclitaxel 10mg/kg

H: Paclitaxel (HS-15) 10mg/kg

Figure 6

Anti-metastasis potency of SY-707 in mouse 4T1 model. Nude mice bearing 4T1 breast cancer was adminstered with SY-707 (100 mg/kg, PO, QD) / paclitaxel (20 or $10 \mathrm{mg} / \mathrm{kg}, \mathrm{IV}, \mathrm{QW}$ ) alone (C, D and G) or combined ( $E$ and $F$ ) for 20 days (6mice/group). Mouse lungs were taken at the last day of treatment and fixed with $4 \%$ paraformaldehyde for 24 hours, then the lungs were photographed and the metastatic nodules were calculated manually for further analysis. ${ }^{*}, p<0.05 ;{ }^{* *}, p<0.01$. 


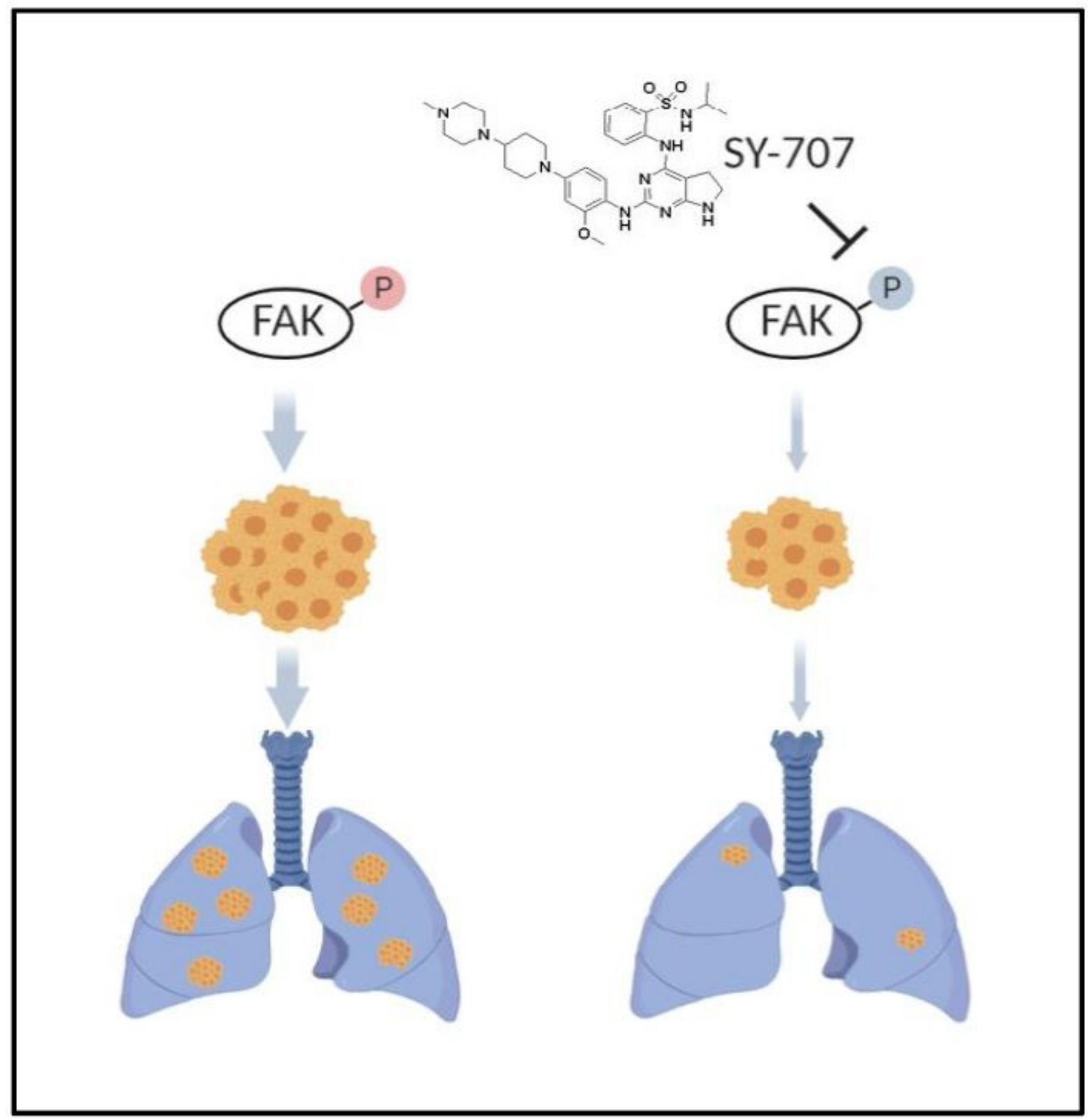

Figure 7

Proposed model of SY707 in inhibiting the phosphorylation of FAK to suppress tumor growth and metastasis.

\section{Supplementary Files}


This is a list of supplementary files associated with this preprint. Click to download.

- originaldata.pptx 